Revista Brasileira de Agricultura Irrigada v.10, nº .5, p. 893 - 903, 2016

ISSN 1982-7679 (On-line)

Fortaleza, CE, INOVAGRI - http://www.inovagri.org.br

DOI: $10.7127 /$ rbai.v10n500459

Protocolo 459.16 - 14/08/2016 Aprovado em 14/10/2016

\title{
ESPACIALIZAÇÃO DA PRECIPITAÇÃO PARA A REGIÃO DO SUBMÉDIO SÃO FRANCISCO
}

\author{
Iug Lopes ${ }^{1}$ Brauliro Gonçalves Leal ${ }^{2}$ Clovis Manoel Carvalho Ramos ${ }^{3}$ Juliana Maria \\ Medrado de Melo ${ }^{4}$
}

\begin{abstract}
RESUMO
Dentre as variáveis meteorológicas requeridas para o cálculo do balanço hídrico destaca-se a precipitação, medida em estações meteorológicas de forma pontual, mas que pode ser convertida em campos contínuos utilizando métodos de interpolação espacial. O objetivo deste trabalho foi avaliar potências para o método de interpolação do Inverso da Potência da Distância (IPD) na espacialização de valores diários da precipitação no Submédio São Francisco, para os períodos de um ano, das estações do ano (inverno, primavera, verão e outono). A precipitação foi espacializada utilizando o método do IPD a partir dos dados medidos em 14 estações meteorológicas automáticas, sendo a área representando cartograficamente através um círculo de aproximadamente $250 \mathrm{~km}$ de raio em torno da cidade de Petrolina-PE. Os valores obtidos da potência do interpolador foram iguais a 1,6, 0,5, 1,0, 3,1 e 0,9 para a época anual, inverno, primavera, verão e outono, respectivamente. Os valores de erro médio foram relativamente pequenos.
\end{abstract}

Palavras-chave: interpolação, estações do ano, estação meteorológica.

\section{SPATIALIZATION OF RAINFALL FOR THE REGION OF THE LOWER MIDDLE SÃO FRANCISCO}

\begin{abstract}
Among the meteorological variables required for calculating the water balance there is the precipitation measured at weather stations in a timely manner, but that can be converted into continuous fields using spatial interpolation methods. The objective of this study was to evaluate powers to the inverse of the interpolation method of Distance Power (IDW) in the spatial distribution of daily values of precipitation in lower middle São Francisco, for periods

${ }^{1}$ Universidade Federal Rural de Pernambuco, Departamento de Engenharia Agrícola, Recife-PE, E-mail: iuglopes@hotmail.com;

${ }^{2}$ Universidade Federal do Vale do São Francisco, Colegiado de Engenharia da Computação, Juazeiro-BA, Email: brauliro.leal@univasf.edu.br;

${ }^{3}$ Universidade Federal do Vale do São Francisco, Colegiado de Engenharia Agrícola, Juazeiro-BA, E-mail: clovis.ramos@univasf.edu.br;

${ }^{4}$ Universidade do Estado da Bahia, Departamento de Agronomia, Juazeiro-BA, E-mail: medrado.juliana@gmail.com
\end{abstract}


of one year of the seasons (winter, spring, summer it's fall). Precipitation was spatialized using IDW method from the data measured at 14 automatic weather stations, and representing cartographically by a circle of about $250 \mathrm{~km}$ radius around the city of Petrolina PE. The values of power interpolating were equal to 1.6, 0.5, 1.0, 3.1 and 0.9 for the year season, winter, spring, summer and fall, respectively. The average error values were relatively small.

Keywords: interpolatiom, seasons, meteorological station.

\section{INTRODUÇÃO}

No Brasil e no mundo, o maior usuário de água é a produção de alimentos através da irrigação, com $69 \%$ do uso destinado a esta finalidade (Braga, 2008). Para se manter sustentável, em termos ambientais, a agricultura irrigada precisa utilizar os recursos hídricos com maior eficiência, o que pode ser alcançado por meio do manejo da irrigação. Uma das formas é o cálculo do balanço hídrico, um método que permite a gestão da água de irrigação, quantificação das necessidades hídricas das culturas em termos de lâmina e duração da irrigação. Isso pode ser feito a partir dos elementos meteorológicos e fatores do solo, da cultura e do sistema de irrigação (Allen et. al., 1998; Peña-Arancibia et. al., 2013).

Dentre as variáveis meteorológicas requeridas para o cálculo do balanço hídrico destaca-se a precipitação, medida em estações meteorológicas de forma pontual, mas que pode ser convertida em campos contínuos utilizando métodos de interpolação espacial (Jakob e Young, 2006). O entendimento do comportamento pluviométrico se faz importante para o dimensionamento de projetos para irrigação e projetos agrícolas, além de que o conhecimento da precipitação por estações do ano faz com que possa ocorrer um planejamento do manejo de água (Tuo et. al., 2016).

Existem métodos de interpolação variados, sendo alguns: Inverso da potência da distância, Krigagem e métodos de vizinhos mais próximos. Métodos geoestatísticos também têm sido utilizados para a espacialização de informações pluviométricas (Gardiman Júnior et al., 2012, Viola et al., 2012).
Um dos procedimentos mais utilizados para avaliar o desempenho destes métodos é o processo de validação cruzada, que permite comparar valores simulados e os valores empíricos obtidos na amostragem, e com base na análise deste erro de estimação pode-se selecionar o melhor modelo (Nesi et. al., 2013). Inverso da potência da distância foi descrito por Willmott e Matsuura (2006), estudando erros de dados meteorológicos interpolados obtidos a partir de observações mensais de 1807 estações meteorológicas da Rede Climatológica Histórica Global, observaram que o Erro Absoluto Médio (EAM) e o Viés Médio (VM) são as medidas mais adequada para avaliar os erros de interpoladores espaciais. Além da área meteorológica, existem várias pesquisas que consolidam a verificação por esses dois métodos (Delgado et al., 2012; Borges Júnior et al., 2012, Mei et al., 2016).

Tendo em vista a importância das variáveis meteorológicas para a gestão de recursos hídricos, o objetivo deste trabalho foi avaliar potências para o método de interpolação do Inverso da Potência da Distância (IPD) na espacialização de valores diários da precipitação da região do Submédio São Francisco, para os períodos de um ano, da estação de inverno, de primavera, de verão e outono.

\section{MATERIAL E MÉTODOS}

\section{Caracterização da área de estudo.}

A área estudada do Submédio São Francisco compreende o Pólo de Desenvolvimento Petrolina-Juazeiro, situado na zona mais árida do Nordeste Brasileiro, às margens do Rio São Francisco, no extremo 
Oeste de Pernambuco e Norte da Bahia. Ele conta com uma área de cerca de 260.000 hectares irrigados (CODEVASF, 2014).

A região de estudo, é representada cartograficamente através um círculo de aproximadamente $250 \mathrm{~km}$ de raio em torno da cidade de Petrolina-PE (Figura 1), no Semiárido Nordestino.

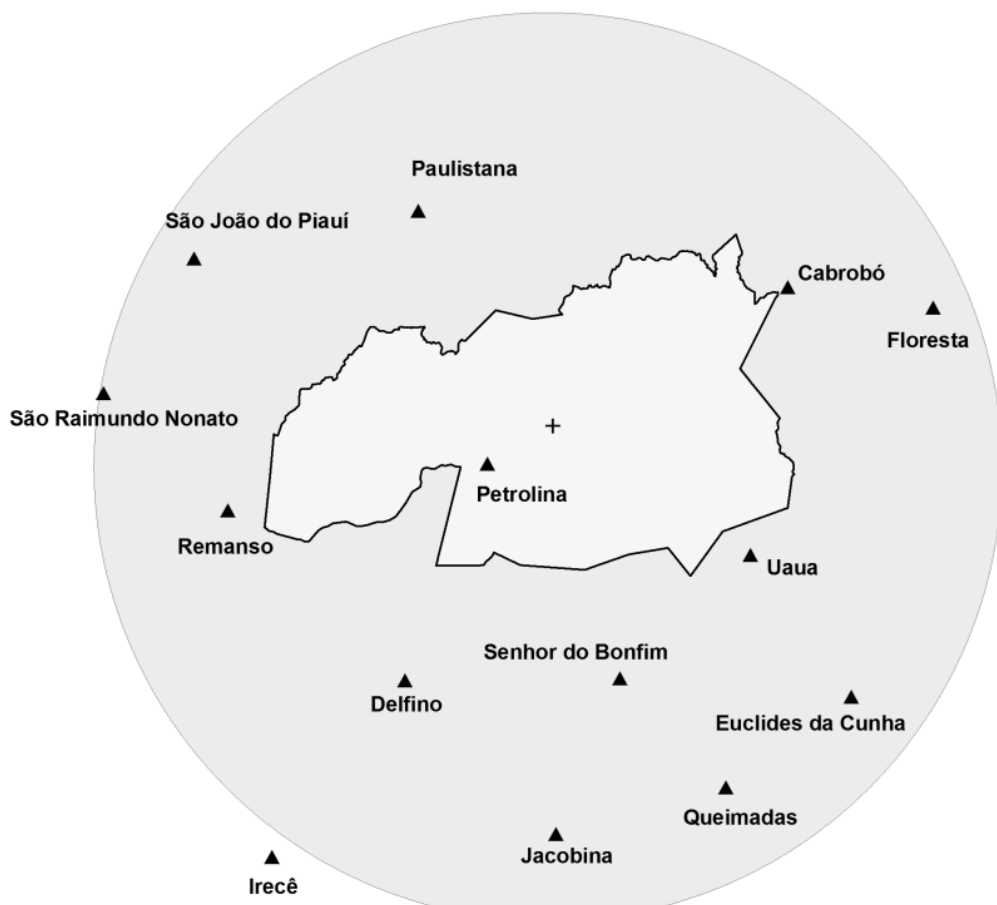

Figura 1. Espacialização das estações automáticas do INMET utilizadas no estudo, com distância de referência da estação de Petrolina-PE.

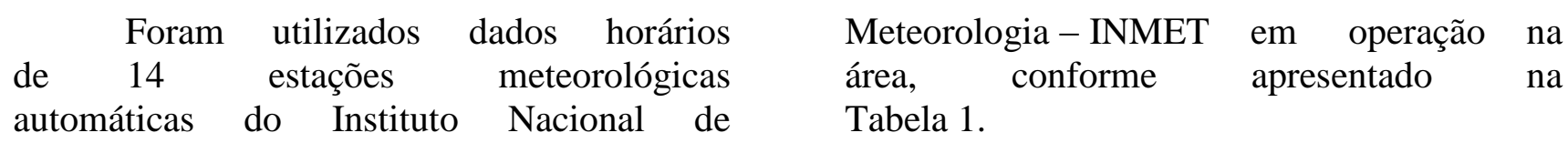

Tabela 1. Estações meteorológicas automáticas do INMET utilizadas no trabalho com código, local, Unidade Federativa (UF), Latitude e Longitude

\begin{tabular}{ccccc}
\hline Código & Local & UF & Latitude $\left(^{\circ}\right)$ & Longitude $\left(^{\circ}\right)$ \\
\hline A329 & Cabrobó & PE & $-9,3833$ & $-40,8012$ \\
A443 & Delfino & BA & $-10,4553$ & $-41,2072$ \\
A442 & Euclides da Cunha & BA & $-10,4442$ & $-40,1469$ \\
A351 & Floresta & PE & $-8,1325$ & $-41,1428$ \\
A424 & Irecê & BA & $-9,6189$ & $-42,0831$ \\
A440 & Jacobina & BA & $-9,8336$ & $-39,4956$ \\
A330 & Paulistana & PI & $-8,5036$ & $-39,3144$ \\
A307 & Petrolina & PE & $-8,3647$ & $-42,2508$ \\
A436 & Queimadas & BA & $-11,205$ & $-40,4653$ \\
A423 & Remanso & BA & $-9,0331$ & $-42,7006$ \\
A331 & São João do Piauí & PI & $-10,9847$ & $-39,6171$ \\
A345 & São Raimundo Nonato & PI & $-10,5367$ & $-38,9978$ \\
A428 & Senhor do Bonfim & BA & $-11,3289$ & $-41,8647$ \\
A435 & Uauá & BA & $-8,6103$ & $-38,5922$ \\
\hline
\end{tabular}


Na Figura 2 pode-se ver em a altitude destas estações e em suas distâncias em relação à estação de Petrolina.

Avaliação dos dados de precipitação

Os valores diários da precipitação foram obtidos a partir dos dados horários medidos nas estações meteorológicas automáticas. Foram utilizadas apenas os dias que possuíam 24 dados horários válidos, organizadas em dois períodos de dados:

- Primeiro período - de 21/06/2008 a 20/06/2009, com cinco épocas, ano (21/06/2008 a 20/06/2009), inverno $\quad(21 / 06 / 2008 \quad a$
22/09/2008), primavera (23/09/2008 a 20/12/2008), verão (21/12/2008 a 20/03/2009) e outono (21/03/2009 a 20/06/2009), estes dados foram utilizados para estimar os valores de p destas épocas consideradas;

- Segundo período - de 21/06/2009 a 21/03/2010, estes dados foram utilizados para avaliar o erro do valor da potência na época de concentração de precipitação da região.

\section{— Distância O Altitude}

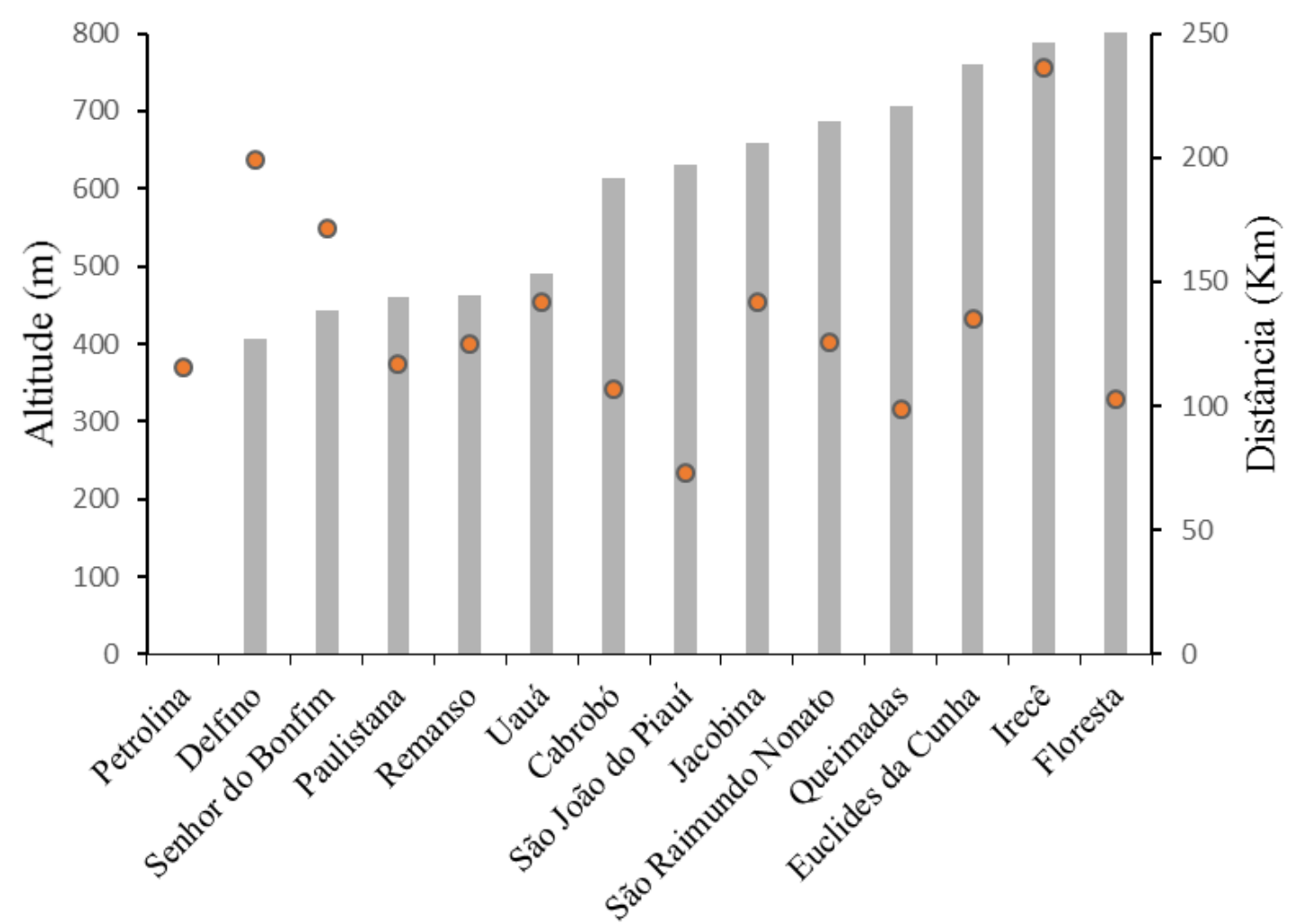

Figura 2. Altitude das estações e em suas distâncias em relação à estação de Petrolina-PE

Para a espacialização das precipitações diárias avaliaram-se as potências para o método de interpolação do Inverso da Potência da Distância (IPD). Variou-se o parâmetro de potência de 0,0 a 25,0, com incremento de 0,1 . Metodologia também usada por Castro et al. (2010) e Silva et al. (2011).

A metodologia do IPD leva em consideração que o quantitativo da variável, a ser estimado numa posição qualquer, é calculado pelos " $n$ " vizinhos mais próximos e assim ponderados pelo inverso da sua distância elevada a uma potência " $p$ ", conforme a Equação 1.

O desempenho do IPD foi determinado utilizando os valores do Erro Absoluto Médio (EAM) (Equação 2) e de Viés Médio (VM) 
(Equação 3), calculados por meio da técnica de Validação Cruzada (VC) aplicada aos dados do primeiro período.

$$
\begin{aligned}
& \mathrm{f}_{\mathrm{e}}(\mathrm{r})=\frac{\sum_{\mathrm{i}=1}^{\mathrm{n}} \mathrm{d}\left(\mathrm{r}, \mathrm{r}_{\mathrm{i}}\right)^{-\mathrm{p}} \mathrm{f}_{\mathrm{m}}\left(\mathrm{r}_{\mathrm{i}}\right)}{\sum_{\mathrm{i}=1}^{\mathrm{n}} \mathrm{d}\left(\mathrm{r}, \mathrm{r}_{\mathrm{i}}\right)^{-\mathrm{p}}} \quad \text { (Equação 1) } \\
& E A M=\frac{1}{n} \sum_{i=1}^{n}\left|f_{m}\left(r_{i}\right)-f_{e}\left(r_{i}\right)\right| \quad \text { (Equação 2) } \\
& V M=\frac{1}{n} \sum_{i=1}^{n}\left[f_{m}\left(r_{i}\right)-f_{e}\left(r_{i}\right)\right] \quad \text { (Equação 3) }
\end{aligned}
$$

em que,

$f_{(r)}$ - valor estimado de $\mathrm{f}$ no vetor posição $r$,

$\mathrm{fm}_{\text {(ri) }}$ - valor medido de $\mathrm{f}$ no vetor posição ri,

$\mathrm{n}$ - número total de pontos conhecidos e utilizados na interpolação,

$\mathrm{d}_{(\mathrm{r}, \mathrm{ri})}$ - distância euclidiana entre os vetores $r$ e ri, e

p - parâmetro de potência.

$\mathrm{Na}$ elaboração da VC, os valores calculados com dados de menos de 13 estações meteorológicas automáticas foram desconsiderados. Nesta etapa foram obtidos os valores da potência das épocas ano, inverno, primavera, verão e outono, a fim de observar se a aplicação da potência da interpolação por estação do ano é mais precisa que a aplicação igual para todo o ano.

Realizou-se a validação cruzada dos dados, segundo a metodologia de Robinson \& Metternicht (2006) e Amorim et al. (2008). Essa metodologia consiste na qual um ponto de medida é descartado sucessivamente na realização da interpolação, sendo possível obter o valor estimado (E) relativo ao retirado e desta forma pode-se compará-lo com o valor real da variável (O) (Piovesan, et al. 2009).
Toda a metodologia descrita foi realizada de forma automatizada, permitindo uma maior garantia e agilidade nas operações.

$\mathrm{O}$ valor de $\mathrm{p}$ da época ano, obtido com os dados do primeiro período, foi avaliado utilizando os dados do segundo período por meio dos valores do erro relativo médio diário, conforme definido a seguir:

- erro relativo de precipitação diária $\left(\mathrm{er}_{\mathrm{dia}}\right)$, o erro relativo calculado a partir dos valores de precipitação diários estimados (Xe) e valores de precipitação diários medidos $\left(\mathrm{X}_{\mathrm{m}}\right)$ em uma estação meteorológica, Equação 4.

- erro relativo médio de precipitação diária ( $\left.\mathrm{er}_{\mathrm{med}}\right), \quad \mathrm{a}$ média aritmética de er $_{\text {dia }}$ de um conjunto de estações meteorológicas, Equação 5.

$$
\begin{aligned}
& e r_{\text {dia }}=\frac{X_{m}-X_{e}}{X_{m}} \quad(\text { Equação 4) } \\
& e r_{\text {med }}=\text { média }\left\{e r_{\text {dia }}\right\} \quad(\text { Equação 5) }
\end{aligned}
$$

\section{RESULTADOS E DISCUSSÃO}

Do primeiro período foram utilizados 232 valores de precipitação, calculados utilizando dados horários medidos nas estações meteorológicas. Os valores mínimo, médio, mediana, máximo, coeficiente de variação $(\mathrm{CV})$, primeiro quartil (Q1/4) e terceiro quartil (Q3/4) dos dados utilizados por época do primeiro período estão apresentadas na Figura 3. O conhecimento da precipitação por estações do ano faz com que possa ocorrer um planejamento trimestral de uso da água para a agricultura. 

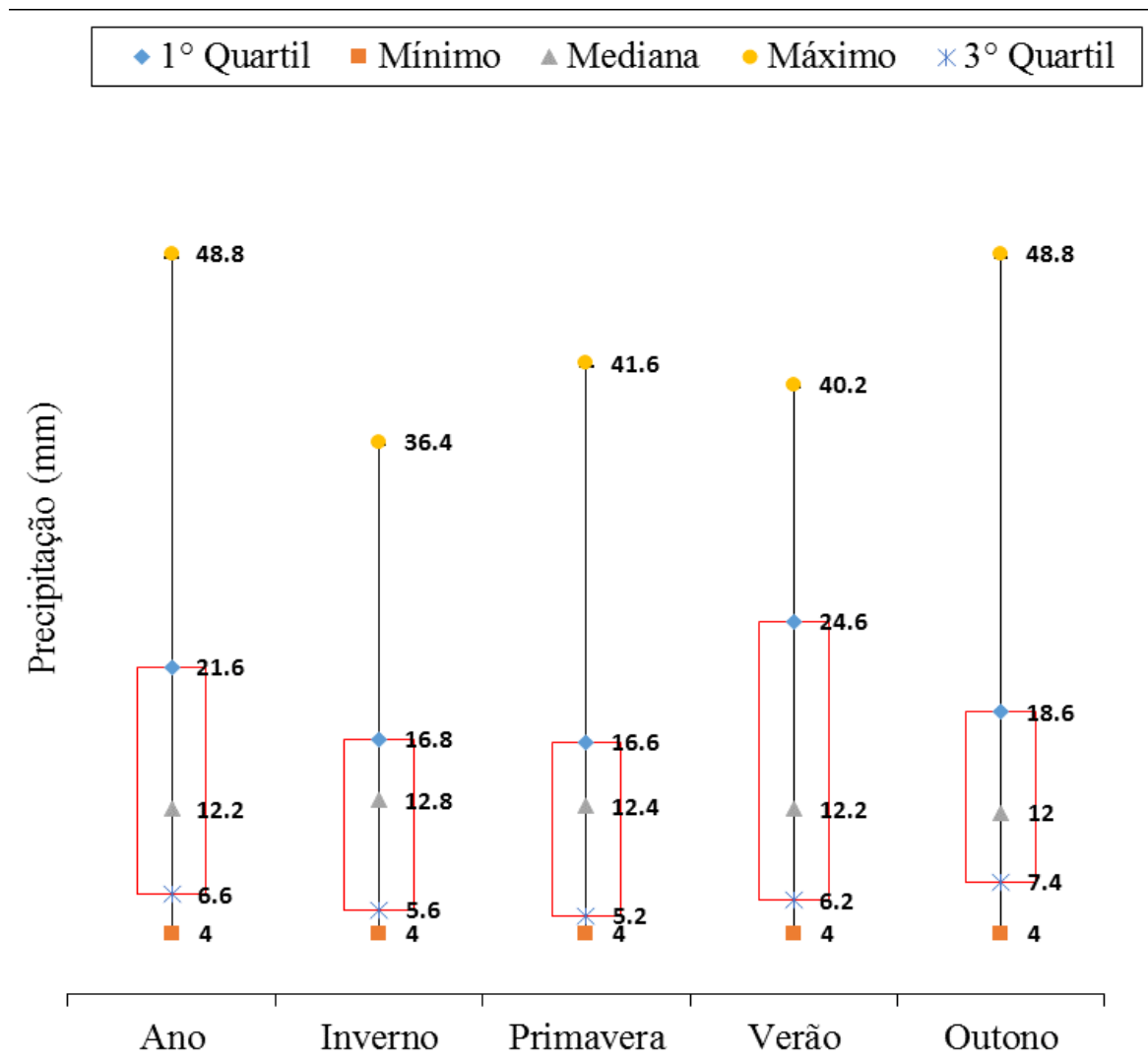

Figura 3. Boxplot dos dados de precipitação utilizados nas épocas ano, inverno primavera, verão e outono do primeiro período.

O desvio padrão e o número de dados utilizados estão apresentados na Tabela 2. Pode-se verificar que a variação do desvio padrão foi pouca entre os períodos e os números de dados utilizados variaram conforme o regime pluviométrico da região.

Tabela 2. Estatísticas de precipitação do primeiro período e suas épocas.

\begin{tabular}{lcr}
\hline \multicolumn{1}{c}{$\begin{array}{c}\text { Primeiro } \\
\text { Período }\end{array}$} & $\begin{array}{c}\text { s } \\
(\mathrm{mm})\end{array}$ & $\mathrm{n}$ \\
\hline ano & 10,40 & 232 \\
inverno & 9,34 & 14 \\
primavera & 12,05 & 14 \\
verão & 10,94 & 93 \\
outono & 9,93 & 111 \\
"S" corresponde ao desvio & padrão e "n" \\
número de dados utilizados. &
\end{tabular}

Pode-se verificar na Figura 3, que os valores de precipitação do primeiro período variaram de 4,0 a 48,8 mm, com desvio padrão e coeficiente de variação igual a 15,1 e 10,40 $\mathrm{mm}$, respectivamente. Em termos anuais, $50 \%$ dos valores de precipitação estão entre 6,6 e
21,6 mm. Os valores médios de precipitação no período estudado de inverno, primavera, verão e outono foram iguais a 13,5, 14,7, 15,9 e $14,7 \mathrm{~mm}$, respectivamente.

Na Figura 4 pode observar a variação de Erro Absoluto Médio (EAM) em função de potência nas épocas e período estudado. A variação do EAM foi similar nas épocas, com uma tendência de valor inicial mais elevado, acompanhado por uma sutil redução até um valor mínimo, seguido de um crescimento moderado.

A curva correspondente ao inverno se destaca das demais, apresentado os maiores valores de EAM. As demais, ano, primavera, verão e outono, apresentam valores similares principalmente próximos dos seus valores mínimos. Este comportamento pode ser devido à topografia da região, em que as altitudes das estações meteorológicas variaram de 235 a $755 \mathrm{~m}$, e esse fator é um dos quais influencia o clima próximo à superfície, como já foi observado por Raupach e Finnigan (1997). A sazonalidade da cobertura vegetal também pode estar reforçando este comportamento, em 
que se observa um grande quantitativo de variação no inverno (SOUZA et al., 2015).

Os valores mínimos de EAM foram $7,54,2,72,8,99,9,95$ e 7,35 $\mathrm{mm}$ para a época anual, inverno, primavera, verão e outono, respectivamente, com os correspondentes valores de p iguais a 1,6, 0,5, 1,0,3,1 e 0,9. De uma maneira geral os resultados obtidos foram dependentes da influência da sazonalidade nas escalas

consideradas.

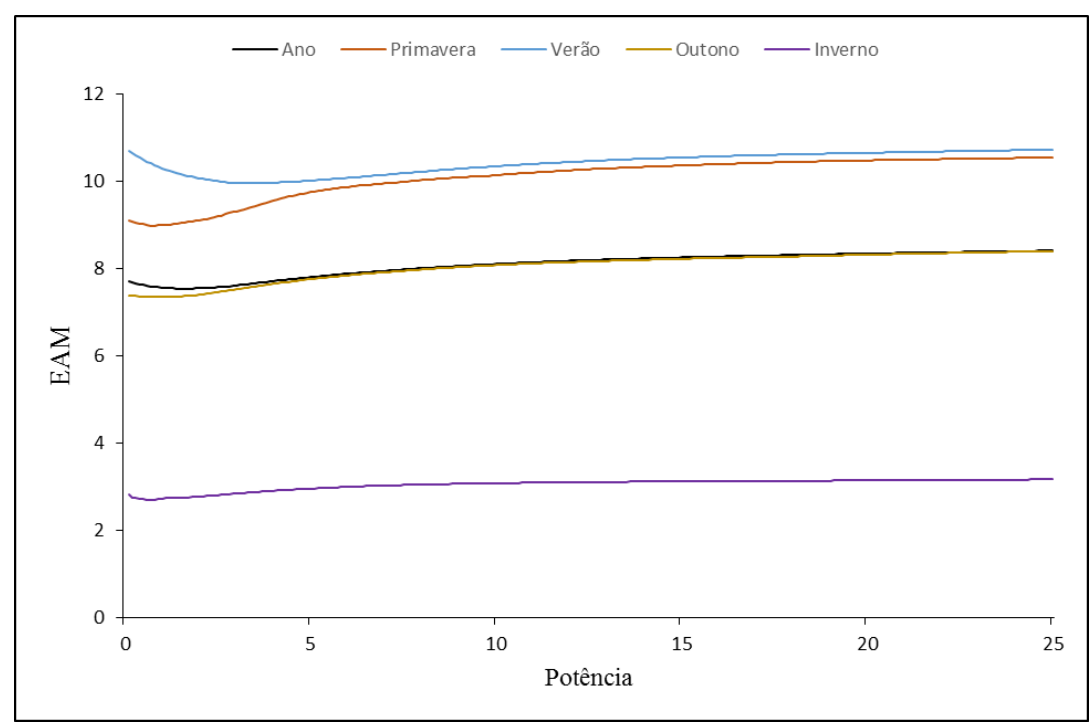

Figura 4. Erro Absoluto Médio para as potências utilizadas e nas épocas ano, inverno primavera, verão e outono do primeiro período.

A Figura 5 apresenta a variação de VM em função de $\mathrm{p}$ nas épocas e período estudado. A variação geral do VM foi similar nas épocas, com valor inicial baixo, acompanhado por um aumento acentuado até um valor máximo, acompanhado por uma diminuição até um valor mínimo local e, em seguida, um crescimento moderado, exceto para a curva correspondente ao inverno que se destaca das demais, apresentado valores crescentes de VM com uma pequena oscilação.

Os valores mínimos de VM foram -0,32, $-0,45,-0,03,-0,03$ e $-0,42 \mathrm{~mm}$ para a época anual, inverno, primavera, verão e outono, respectivamente, sendo os correspondentes valores de $p$ iguais a 3,1, 25,0, 0,1, 10,1 e 2,6 (Tabela

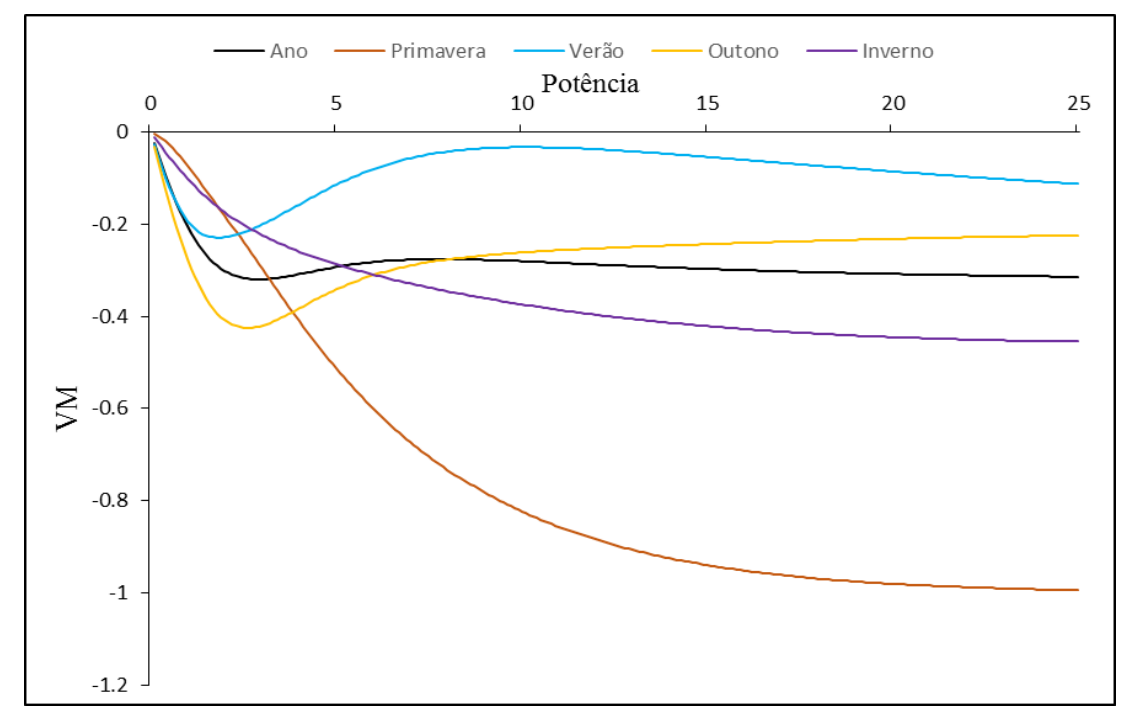

Figura 5. Viés Médio para as potências utilizadas e nas épocas ano, inverno primavera, verão e outono do primeiro período. 
Apesar de existir uma maior tendência da utilização do método Krigagem para dados de precipitação, há uma variação entre as diversas regiões do País. Em regiões com uma maior variação espaço-temporal, apresenta melhor desempenho para interpolação do método IPD (Amorim et al., 2008). Sendo esta característica observada na região em estudo.

A comparação do comportamento de EAM e VM, permite inferir que EAM, por ter um comportamento semelhante para as épocas e também por apresentar um único ponto de mínimo, é a melhor medida de desempenho para avaliar os erros médios de interpolação espacial de precipitação na região e período estudado, o que está de acordo com o resultado obtido por Willmott e Matsuura (2006).

Na Figura 6 consta a variação diária da precipitação medida e estimada utilizando os dados do segundo período e valor de $\mathrm{p}$ obtido para a época ano, precipitação igual a 1,6. Pode-se observar a grande concordância entre os valores medidos e estimados de precipitação utilizando os dados do segundo período.

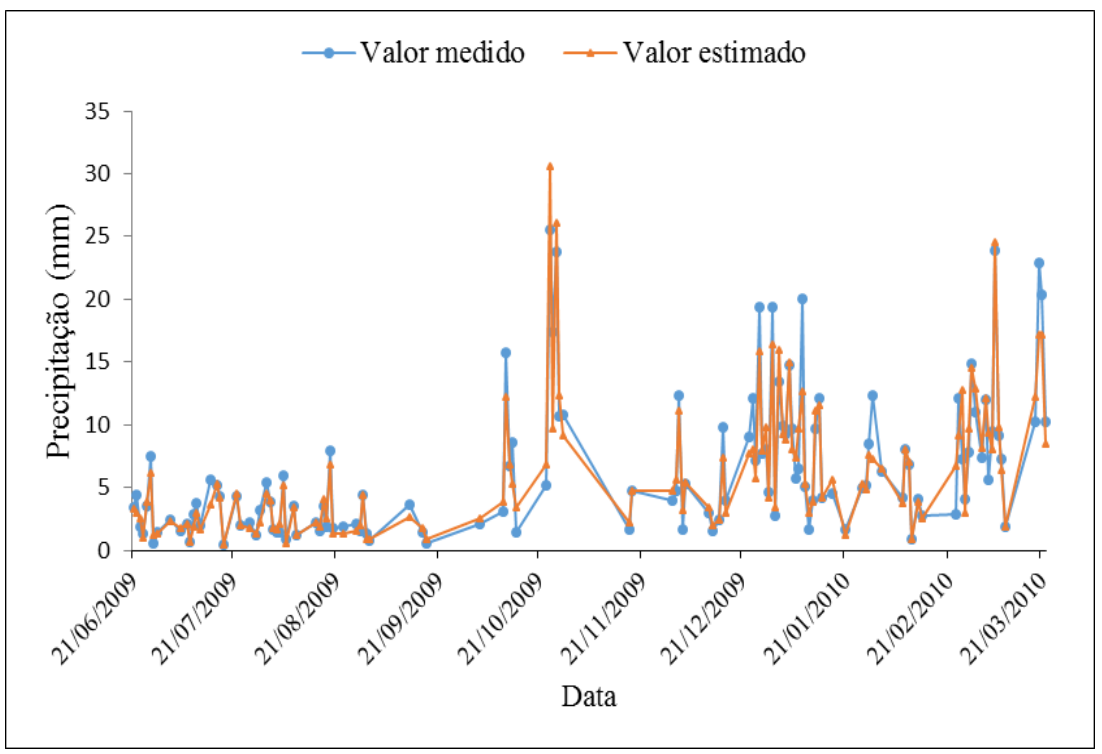

Figura 6. Precipitação medida e estimada para o segundo período.

De modo geral os valores de $\mathrm{er}_{\text {med }}$ foram relativamente pequenos (Figura 7), variando de -39 a $37 \%$, visto que a precisão do instrumento de medição da precipitação em estações automáticas ser de 5\% (Vaisala, 2011).

Em uma análise mais detalhada para os valores de precipitação acima de $15 \mathrm{~mm}$, os valores médios de erro foram de $-8 \%$, com uma variação máxima de -19 a $14 \%$. Assim pode-se inferir que quanto maior a precipitação diária nas localidades da estação, menores os erros associados a interpolação pelo Inverso da Potência da Distância a uma $p$ de 1,6. Observa-se também que nessa metodologia há uma tendência de subestimativa da precipitação para valores acima de $15 \mathrm{~mm}$.

Esse resultado diferente da tendência descrita por Cecílio et al. (2009) para estações do estado do Espírito Santo, onde foi observado que à medida que eleva a precipitação, os erros médios de estimativa também são aumentados e varia entre $14,5 \%$ e $42,5 \%$.

A metodologia se mostra precisa para análise de espacialização de precipitação, em que estes dados interpolados já foram utilizados para espacialização das erosões no estado de Minas Gerais (Menezes e Leite, 2011) e para fins de elaboração do Balanço Hídrico Climatológico (Cecílio, 2012). 


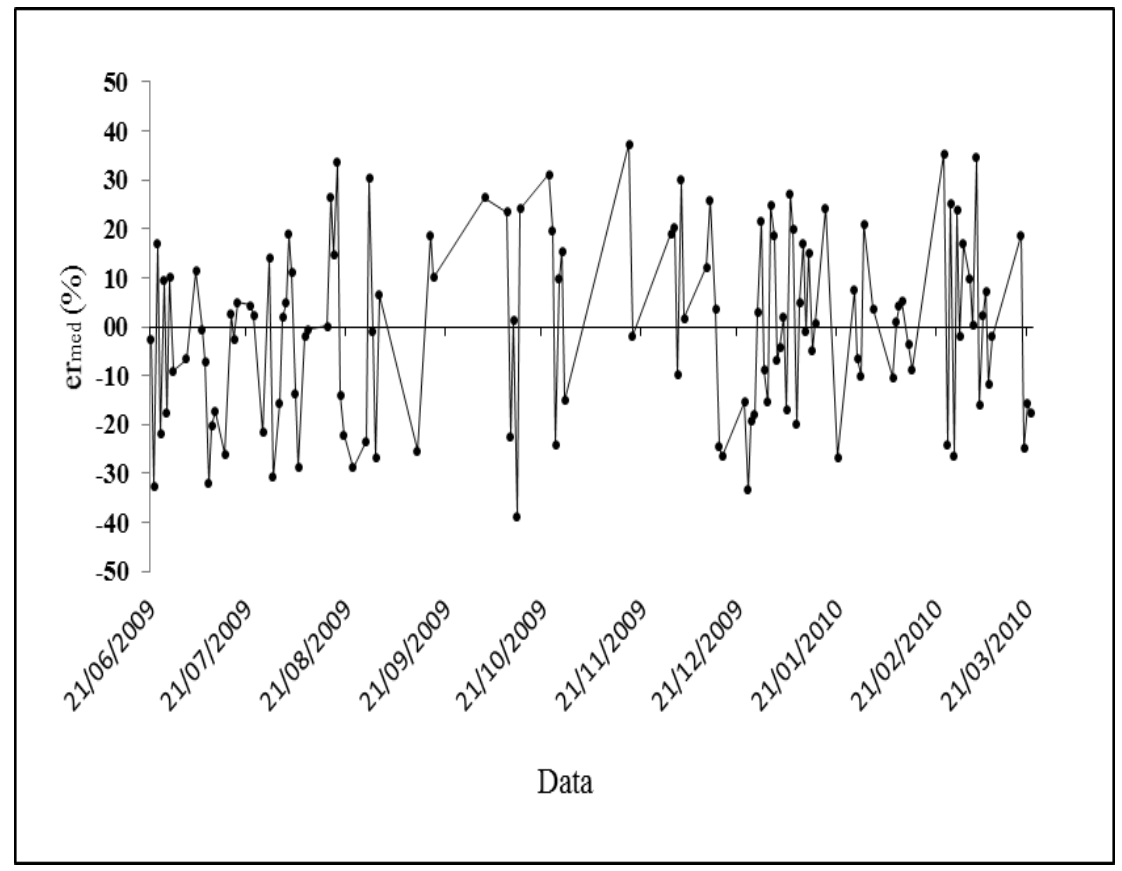

Figura 7. Erro médio entre precipitação medida e estimada para o segundo período.

Os valores dos erros encontrados neste trabalho foram menores do que aqueles encontrados por Castro et al. (2010) e sua sazonalidade podem estar relacionados ao clima da região que apresenta períodos secos e chuvosos bem definidos bem como à sua topografia. O Lago de Sobradinho, localizado na área de estudo, pode também estar exercendo influência na precipitação da região como é apontado por Correia \& Dias (2003).

\section{CONCLUSÕES}

Foram estimados os valores da potência (p) do inverso da potência da distância para a interpolação da precipitação da região, utilizando validação cruzada e erro absoluto médio. Os valores de $\mathrm{p}$ obtidos foram iguais a $1,1,2,3,1,0,1,1$ e 0,1 para a época ano, inverno, primavera, verão e outono, respectivamente.

O erro absoluto médio foi a melhor medida de desempenho do inverso da potência da distância para a espacialização da precipitação na região e período estudado.

O valor da potência da época ano foi avaliado utilizando o erro relativo diário médio que apresentou valor pequeno, o que viabiliza o método do inverso da potência da distância para a espacialização da precipitação da região estudada.

Estudos devem ser realizados a maximizar o conhecimento da espacialização da chuva, a fim de tornar um subsidio para auxílio na tomada de decisões na irrigação pelos agricultores nos perímetros irrigados que estão presentes nessa e em outras áreas que possuem poucas estações disponíveis.

\section{REFERÊNCIAS BIBLIOGRÁFICAS}

ALLEN, R. G.; PEREIRA, L. S.; RAES, D.; SMITH, M. Crop evapotranspiration Guidelines for computing crop water requeriments. Rome: FAO - Irrigation and drainage, Paper 56, 1998. 319 p.

AMORIM, RANIÉRI CARLOS FERREIRA; RIBEIRO, ARISTIDES; LEAL, BRAULIRO GONÇALVES. Avaliação do comportamento de variáveis meteorológicas espacializadas em áreas de relevo ondulado na bacia do Rio Doce. Engenharia na Agricultura, v. 16(1) p. 19-26, 2008.

BORGES JÚNIOR, J. C. F.; ANJOS, R. J.; SILVA, T. J. A.; LIMA, J. R. S.; ANDRADE, C. L. T. Métodos de estimativa da 
evapotranspiração de referência diária para a microrregião de Garanhuns, Pe. Revista brasileira de engenharia agrícola e ambiental, v.16, p.380-390, 2012.

BRAGA, P. F. Gestão de águas no Brasil. Scientific American Brasil, Março 2008, n.70, p.38-41.

CORREIA, M. DE F.; DIAS, M. A. F. DA S. Variação do Nível do Reservatório de Sobradinho e seu Impacto Sobre o Clima da Região. Revista Brasileira de Recursos Hídricos Volume 8 n.1 Jan/Mar 2003, p157168.

CASTRO, F. DA S.; PEZZOPANE, J.E.M.; CECÍLIO, R.A.; PEZZOPANE, J.R.M.; XAVIER, A.C. Avaliação do desempenho dos diferentes métodos de interpoladores para parâmetros do balanço hídrico climatológico. Revista Brasileira de Engenharia Agrícola e Ambiental, Campina Grande, v.14, p.871-880, 2010.

CECÍLIO, R. A.; SILVA, K. R.; XAVIER A. C. X.; PEZZOPANE J. R. M. Método para a espacialização dos elementos do balanço hídrico climatológico. Pesquisa Agropecuária Brasileira, Vol.47(4), pp.478488, 2012.

CECÍlIO, R. A.; XAVIER, A. C.; PRUSKI, F. F.; HOLLANDA, M. P.; PEZZOPANE, J. E. M. Avaliação de interpoladores para os parâmetros das equações de chuvas intensas no Espírito Santo. Ambi-Agua, Taubaté, v.4, n. 3, p. 82-92, 2009.

CODEVASF-Companhia de Desenvolvimento do Vale do São Francisco e Parnaíba. Projetos de Irrigação no Vale do São Francisco. Brasil, 2014.http://www.codevasf.gov.br/principal/pu blicacoes/publicacoes-atuais/pdf/projetos-deirrigacao-no-vale-do-sao-franciscodocumento-elaborado-pelos-tecnicos-dacodevasf.pdf. Acesso em 26/11/2015.

DELGADO, R. C.; SEDIYAMA, G. C.; COSTA, M. H.; SOARES, V. P.; ANDRADE,
R. G. Classificação espectral de área plantada com a cultura da cana-de-açúcar por meio da árvore de decisão. Revista Engenharia Agrícola, Jaboticabal, v. 32, n.2, p.369-380, mar./abr. 2012.

GARDIMAN JÚNIOR, B.S.; MAGALHÃES, I.A.L.; FREITAS, C.A.A.; CECÍLIO, R.A. Análise de técnicas de interpolação para espacialização da precipitação pluvial na bacia do rio Itapemirim (ES). Ambiência, Guarapuava, v.8, n.1, p.61-71, 2012.

INMET. Monitoramento das Estações Automáticas. Disponível em: $<$ http://www.inmet.gov.br/sonabra/maps/auto maticas.php>. Acesso em 26 jul. 2009, 21:00:00.

JAKOB, A. A. E.; YOUNG, A. F. O uso de métodos de interpolação espacial de dados nas análises sociodemográficas. In: XV Encontro Nacional da Associação Brasileira de Estudos Populacionais, 2006, Caxambu. Anais do XV Encontro Nacional da Associação Brasileira de Estudos Populacionais, 2006.

Mei, G.; Xu, N.; Xu, L. Improving GPUaccelerated Adaptive IDW Interpolation Algorithm Using Fast kNN Search. SpringerPlus, v.5, p.1-22, 2016.

MENEZES, M. D.; LEITE, F. P. Avaliação e espacialização da erosividade da chuva no Vale do Rio Doce, região centro-leste de Minas Gerais. Revista Brasileira de Ciência do Solo, v.34, p.1029 - 1039, 2011.

NESI, C. N.; RIBEIRO, A.; BONAT, W. H.; RIBEIRO J. R., P.J. Verossimilhança na seleção de modelos para predição espacial. Revista Brasileira de Ciência do Solo, Viçosa-MG, v. 37, n.2, p. 352-358, 2013.

PEÑA-ARANCIBIA J. L., VAN DIJK A. I. J. M., RENZULLO L. J., MULLIGAN M. Evaluation of precipitation estimation accuracy inreanalyses, satellite products, and an ensemble method for regions in Australia and South and East Asia. 
Journal Hydrometeorol, v. 14, p. 1323-1333, 2013.

PIOVESAN, PAMELA; ARAUJO, LUCIO BORGES DE; DIAS, CARLOS TADEU DOS SANTOS. Validação cruzada com correção de autovalores e regressão isotônica nos modelos de efeitos principais aditivos e interação multiplicativa. Cienc. Rural [online]. 2009, vol.39, n.4, pp. 1018-1023. Epub 24-Abr2008. ISSN 0103-8478.

RAUPACH, M. R.; FINNIGAN, J. J. The influence of topography on meteorological variables and surface-atmosphere interactions. Journal of Hydrology, 190, p. 182-213. 1997.

SILVA, K.R ; CECILIO, R. A. ; XAVIER, A. C. ; PEZZOPANE, J. R. M. ; GARCIA, G. O. - Interpolação Espacial da Precipitação no Estado do Espírito Santo. FLORAM - Revista Floresta e Ambiente, v. 18, p. 417-427, 2011.

SOUZA, B. I.; MENEZES, R.; ARTIGAS, R. C. Efeitos da desertificação na composição de espécies do bioma Caatinga, Paraíba/Brasil. Investigaciones Geográficas - Instituto de Geografía. Universidad Nacional Autónoma de México, v. 1, p. 45-59, 2015.
TUO, Y.; DUAN, Z.; DISSE, M.; CHIOGNA, G. Evaluation of precipitation input for SWAT modeling in Alpine catchment: A case study in the Adige river basin (Italy). Science of The Total Environment, v. 20; p. 66-82, 2016.

VAISALA. Transmissor climático Vaisala WXT520. Disponível em: <http://br.vaisala.com/br/products/multiweathe rsensors/Pages/WXT520.aspx>. Acesso em: 23 Jul. 2011.

VIOLA, M. R.; MELLO, C. R. de.; PINTO, D. B. F.; MELlO, J. M. de.; ÁVILA, L. F.; Métodos de interpolação espacial para o mapeamento da precipitação pluvial. Revista Brasileira de Engenharia Agrícola e Ambiental, Campina Grande, v.14, n.9, p.970-978, 2012.

WILLMOTT, C. J.; MATSUURA, K. On the use of dimensioned measures of error to evaluate the performance of spatial interpolators. International Journal of Geographical Information Science, Vol. 20, No. 1, January, 89-102, 2006.

WILLMOTT, C. J. On the validation of models. Physical Geography, v.2, p.184-194, 1981. 